\title{
Abordaje subvasto versus parapatelar medial en la artroplastia total de rodilla, resultados funcionales
}

\author{
Subvastus versus medial parapatellar approach in \\ total knee arthroplasty, functional results \\ Jesús Matehuala García, * José Manuel Pérez Atanasio, ${ }^{*}$ \\ Francisco Javier Villarreal Barrera, * Mauricio Quitzé Ramos Gómez ${ }^{\star}$ \\ ${ }^{*}$ Cirujano Ortopédico adscrito al Servicio de Reemplazo Articular del Hospital General Regional No. 2 Villa Coapa, Instituto \\ Mexicano del Seguro Social, Ciudad de México, México; ‡Cirujano Ortopédico adscrito al Servicio de Traumatología del Hospital \\ General Regional No. 2 El Marqués, Instituto Mexicano del Seguro Social, Querétaro, México.
}

\begin{abstract}
Resumen
El reemplazo total de rodilla es en general una cirugía exitosa con una baja incidencia de complicaciones. A pesar de esto, 20\% de los pacientes no están satisfechos con el resultado clínico, lo que probablemente está relacionado con el abordaje quirúrgico, por tal motivo buscamos opciones menos agresivas para la colocación de la prótesis. La capsulotomía parapatelar medial per se es traumática, por tanto, una alternativa viable es incidir a través de los intervalos musculares y el abordaje subvasto es el epítome de esta decisión. Material y métodos: El objetivo de este estudio fue evaluar los resultados funcionales del abordaje subvasto vs. parapatelar medial en la artroplastia total de rodilla. Se incluyeron 64 pacientes con diagnóstico de gonartrosis primaria. Los pacientes fueron evaluados con el Knee Society Score a las cuatro semanas y a los seis meses. Resultados: Del total de 64 pacientes incluidos en el estudio, se distribuyeron en dos grupos. Los sometidos al abordaje parapatelar medial (grupo I) con 32 pacientes y el abordaje subvasto (grupo II) con 32 pacientes. Los resultados mostraron mejor evolución en las primeras cuatro semanas con el abordaje subvasto, pero tras la revisión de los seis meses los resultados fueron muy semejantes. Conclusiones: El abordaje subvasto ofrece un mejor rango de movilidad inicial y menos dolor en las primeras semanas; sin embargo, los resultados son semejantes al compararlos con el abordaje parapatelar medial a mediano plazo.
\end{abstract}

Palabras clave: Abordaje parapatelar, abordaje subvasto, artroplastia de rodilla.

\begin{abstract}
Total knee replacement is generally a successful surgery with a low incidence of complications, despite this $20 \%$ of patients are not satisfied with the clinical result, probably related to the surgical approach, for this reason we look for less aggressive options for the placement of the prosthesis. Medial parapatellar capsulotomy per se is traumatic, therefore, a viable alternative is to incise through the muscular intervals, and the subvastous approach is the epitome of this decision. Material and methods: The objective of this study was to evaluate the functional results of the subvastous vs medial parapetellar approach in total knee arthroplasty. 64 patients with a diagnosis of primary knee osteoarthritis were included. The patients were evaluated with the Knee Society Score at four weeks and six months. Results: Of the total of 64 patients included in the study, they were divided into two groups. Patients underwent the medial parapatellar approach (group I) with 32 patients and the subvastus approach (group II) with 32 patients. The results showed better evolution in the first four weeks with the subvastus approach, but after the six-month revision the results were very similar. Conclusions: The subvastous approach offers a better initial range of motion and less pain in the first weeks, however the results are similar when compared to the medial parapatellar
\end{abstract} approach in the medium term.

Keywords: Parapatellar approach, subvastus approach, knee arthroplasty.

Correspondencia:

Jesús Matehuala García

E-mail: jesus_matehuala_garcia@yahoo.com.mx

Recibido: 06-07-2021. Aceptado: 15-07-2021.
Citar como: Matehuala GJ, Pérez AJM, Villarreal BFJ, Ramos GMQ. Abordaje subvasto versus parapatelar medial en la artroplastia total de rodilla, resultados funcionales. Orthotips. 2021; 17 (4): 206-210. https://dx.doi.org/10.35366/102218 


\section{Introducción}

El número anual de artroplastias totales de rodilla (ATR) se ha incrementado alrededor del mundo en las últimas décadas; es un procedimiento con una técnica reproducible y eficaz, ${ }^{1}$ esta tendencia se entiende debido al éxito del procedimiento, el cual pretende mejorar la función, disminuir el dolor y mejorar la calidad de vida.

La incidencia de complicaciones asociadas a la cirugía es menor de $4 \%$ y a pesar de los beneficios del procedimiento casi $20 \%$ de los pacientes no están totalmente satisfechos con el resultado; puede persistir dolor residual y algunas limitaciones en la flexoextensión así como restricciones físicas en pacientes activos, tales resultados están asociados con factores atribuibles a la técnica, principalmente el abordaje quirúrgico, el diseño del implante, la selección del paciente así como el manejo de las expectativas. $^{2}$

Tras una ATR algunos pacientes pueden experimentar dolor y disminución de la fuerza del cuádriceps, ${ }^{3}$ lo cual se atribuye al abordaje quirúrgico, que de manera habitual se realiza a través de una incisión cutánea longitudinal en la línea media de 20 a $25 \mathrm{~cm}$. Posterior a la elección del cirujano, basándose en una planificación preoperatoria, se lleva a cabo una capsulotomía parapatelar medial, lateral, subvasto o midvasto.

Tradicionalmente el abordaje que se utiliza en la ATR es el parapatelar medial (PPM), la enseñanza del mismo se transmite de un cirujano a otro, lo cual favorece su expansión. La descripción original se atribuye a Von Langenbeck, se realiza a partir de una disección entre el tendón del cuádriceps y el vasto medial, continúa la artrotomía a lo largo de la

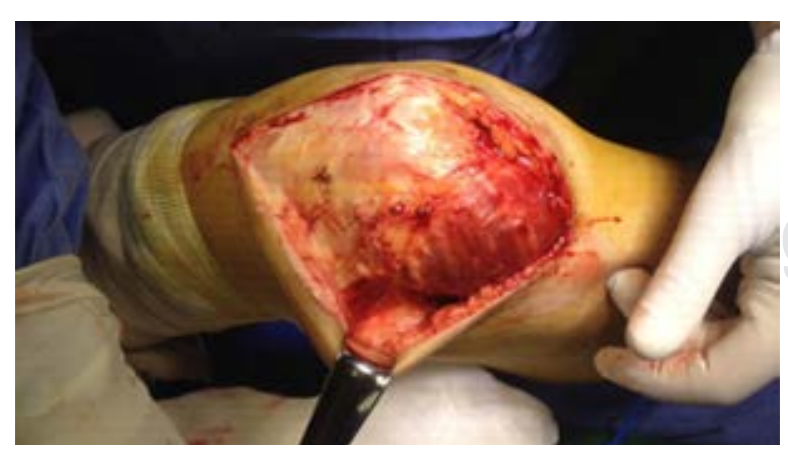

Figura 1: Exposición del vasto medial hasta el canal de los aductores, visualizando el retináculo medial.



cara medial de la rótula; Insall involucra el tendón del cuádriceps y realiza la artrotomía lo más recto posible para evitar seccionar las fibras longitudinales del aparato extensor. ${ }^{4}$ Este abordaje ofrece excelente exposición, es reproducible y versátil, las complicaciones femorales o tibiales son escasas; sin embargo, vulnera el mecanismo extensor y el aporte vascular de la rótula, ${ }^{5}$ lo que propicia la aparición de complicaciones hasta en $12 \%$ como incremento en la inclinación patelar, subluxación, luxación o necrosis avascular. ${ }^{6}$

El abordaje subvasto (SV) fue descrito por Erkes en 1929 y popularizado por Hoffman en 1991, quien concluye que «el abordaje subvasto para la artroplastia total de rodilla primaria es la elección más natural y lógica en comparación con el abordaje parapatelar medial, considerando la anatomía y fisiología, muscular y vascular». ${ }^{7}$ El abordaje en planos profundos en un inicio expone el vasto interno, posteriormente se identifica el septum intermuscular, a partir de este momento se realiza una disección digital lo más proximal posible respetando las estructuras del canal de Hunter distalmente hasta el retináculo medial (Figura 1). Se localizan y ligan los vasos perforantes, a partir de una forma de $L$ se dirige distalmente paralelo al tendón patelar (Figura 2).

Tiene la ventaja teórica de preservar el mecanismo extensor (Figura 3), lo cual se traduce en una mejor fuerza en el cuádriceps, conserva el aporte vascular de la rótula, centraliza el recorrido patelar, hay menos complicaciones en la herida, acelera la rehabilitación, 


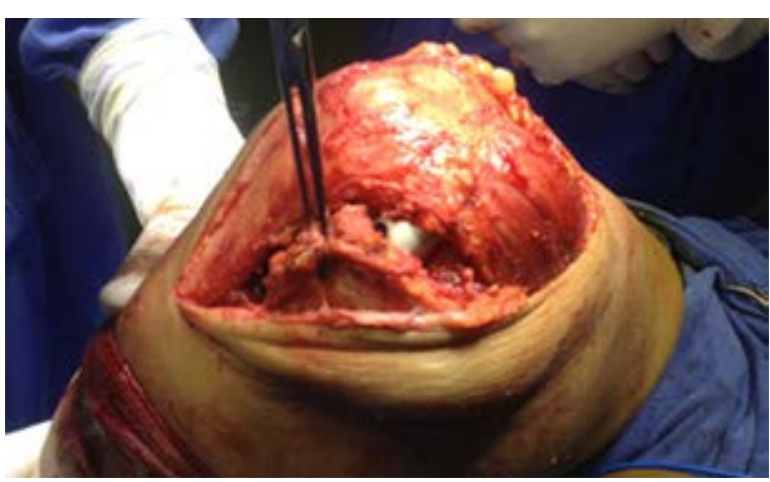

Figura 3: Se observa la integridad del cuádriceps, lo cual mejora el recorrido patelar.

reduce el dolor postoperatorio y disminuye los días de hospitalización. ${ }^{8}$

Sin embargo, la limitante de no evertir la rótula puede restringir la adecuada exposición de la articulación. Algunas contraindicaciones para el uso de este abordaje son: cirugía de revisión, gonartrosis severa, deformidades en valgo, osteotomías alineadoras periarticulares previas y pacientes obesos (Figura 4).

A pesar de las ventajas aparentes y los hallazgos obtenidos con mejores resultados clínicos en múltiples artículos, el abordaje subvasto no destaca como un abordaje que se utilice comúnmente en el reemplazo total de rodilla primario. $Y$ esto puede estar condicionado a la limitada práctica al ejecutar la cirugía con este abordaje.

En el presente estudio evaluamos los posibles beneficios como menos dolor postoperatorio y una mejor recuperación funcional con el abordaje subvasto en comparación con los resultados del abordaje parapatelar medial.

\section{Material y métodos}

Realizamos un estudio prospectivo, el cual incluyó 64 pacientes con diagnóstico de gonartrosis primaria en varo candidatos a ATR, con un índice de masa corporal menor de 35, todos con protocolo preoperatorio completo, los cuales se sometieron a reemplazo total de rodilla primaria utilizando la prótesis NEXURY por Arthronix, con retención del ligamento cruzado posterior y reemplazo de superficie patelar, en ningún procedimiento se colocó torniquete ni se utilizaron antifibrinolíticos, se emplearon antibióticos y antitrombóticos profilácticos, se ejecutaron los dos abordajes quirúrgicos de acuerdo con la descripción original subvasto por Hoffman y parapatelar medial Insall, ambos utilizados de forma aleatoria previa planificación preoperatoria, en ambos abordajes se empleó la misma incisión cutánea, las cirugías fueron realizadas por el mismo equipo quirúrgico en el Hospital Regional No. 2 del IMSS en el período comprendido de Enero a Septiembre de 2019. Se evaluó el tiempo de ejecución de la cirugía así como el sangrado cuantificado por el servicio de anestesiología.

Los pacientes fueron evaluados a las cuatro semanas y a los seis meses, la evaluación funcional se efectuó con el Knee Society Score (KSS), el cual evalúa dolor, estabilidad y rango de movilidad, donde cero es el peor puntaje y 100 el mejor. Además, para el análisis estadístico se empleó la t de Student para variables independientes y de $\chi^{2}$ para la comparación de los dos grupos.

Los resultados fueron expresados como media \pm desviación estándar (DE). Para todas las pruebas se aceptó un valor de significación inferior a 0.05 .

\section{Resultados}

Del total de 64 pacientes incluidos en el estudio, se distribuyeron en dos grupos. El abordaje parapa-



Figura 4: La desventaja parcial de no evertir la rótula es limitar la exposición del compartimento lateral. 
telar medial (grupo I) se efectuó en 32 pacientes y el abordaje subvasto (grupo II) en 32.

Del universo estudiado, 21 fueron hombres (32.4\%) y 43 mujeres $(67.1 \%)$; en el grupo I se presentaron ocho hombres y 24 mujeres, y en el grupo II, 13 hombres y 19 mujeres.

Destacamos que las variables preoperatorias tienen una $p>0.05$, lo que nos habla de que los dos grupos de estudio son similares y confirma la homogeneidad de los dos grupos que buscábamos con nuestra metodología de estudio.

El grupo I presentó una media de edad de 69.93 años (rango: de 55 a 87 ) y el grupo II una media de edad de 67.12 años (rango de 54 a 85).

El abordaje parapatelar fue de tres minutos en promedio más rápido que el abordaje subvasto con una confiabilidad de $95 \%$. Promedio $64.03 \pm 4.7755$ min, mientras que en el abordaje SV el promedio fue $67.28 \pm 6.9988 \mathrm{~min}$, con una $p=0.806$.

En cuanto al sangrado, el abordaje SV mostró un sangrado menor en promedio de $15 \mathrm{ml}$ que el abordaje PPM, lo cual no representa una variación significativa entre ambos abordajes, con el parapatelar (PP) el promedio fue $225.94 \pm 55.1748 \mathrm{ml}$, mientras que el abordaje SV tuvo un promedio de $209.38 \pm 61.3787$ $\mathrm{ml}$, con una $\mathrm{p}=0.261$.

El dolor evaluado a seis meses es menor con la técnica de abordaje SV que con el PP con $95 \%$ de confiabilidad.

No hubo una diferencia significativa en el rango de flexión a cuatro semanas o seis meses entre los dos abordajes. Ambos abordajes evaluados a cuatro semanas presentan un rango de flexión menor de $100^{\circ}$ y a seis meses mayor de $120^{\circ}$.

\section{Discusión}

Se han descrito múltiples abordajes quirúrgicos para la sustitución protésica de la articulación de la rodilla, todos ellos diseñados para permitir el mejor acceso dependiente de la deformidad mecánica 0 indicación específica. A pesar del conocido éxito del procedimiento, la presencia del dolor postoperatorio desencadena la polémica de ¿cuál es el abordaje óptimo para el reemplazo total de rodilla?

El éxito de cualquier procedimiento quirúrgico involucra el conocimiento de la anatomía, la disección de los tejidos implica un daño controlado que repercute no sólo en la sección de estructuras vasculares arteriales o venosas, sino también en la modificación de la fuerza de grupos muscu- lares y en forma secundaria su comportamiento biomecánico.

Cuatro músculos en la cara anterior del muslo forman el cuádriceps: el recto femoral, el crural, el vasto lateral y el vasto medial; la función principal de este grupo muscular es la extensión de la rodilla. El cuádriceps comparte un tendón común de inserción, las fibras del recto anterior y del crural se insertan de manera casi perpendicular en el polo superior de la rótula, las fibras del vasto externo se dirigen oblicuamente en un ángulo de $14^{\circ}$ (entre $6^{\circ}$ y $45^{\circ}$ ) y las fibras del vasto interno a $55^{\circ}$ (entre $28^{\circ}$ y $70^{\circ}$ ). En 1968 Lieb y Perry describieron dos segmentos bien definidos del vasto medial, proximalmente una porción denominada vasto medialis longus (VML), la cual contribuye de manera directa a la extensión de la rodilla, y una porción distal referida como el vasto medial oblicuo (VMO) que actúa como estabilizador medial de la rótula durante la extensión de la rodilla. ${ }^{9}$

Scapinelli analizó el patrón vascular de la rótula basado en la prueba de perfusión de Trueta y Harrison, ${ }^{10}$ sus hallazgos mostraron la presencia de dos sistemas, uno penetra en el tercio medio de la superficie anterior y el otro entra en el polo inferior del hueso detrás del ligamento rotuliano. El patrón arterial extraóseo se caracteriza por la presencia de un anillo altamente anastomótico formado por cinco arterias geniculares.

El abordaje parapatelar medial es considerado el estándar de oro, su popularidad se fundamenta en la sencilla ejecución de cirugía primaria y de revisión. Es útil en cualquier deformidad y la capacidad de exposición facilita la liberación de adherencias que se presentan en contracturas severas. Los problemas surgen cuando se vulnera el complejo extensor, al seccionarse el tendón se sacrifica la biomecánica del mismo y la irrigación de la rótula, ${ }^{11}$ incrementando las complicaciones femoropatelares. Al realizar un abordaje parapateral medial se interrumpe el flujo de las arterias suprema y genicular inferior, obligando a que la integridad de la arteria lateral superior preserve el aporte vascular; el paso crítico de una liberación del retináculo lateral para mejorar el seguimiento patelar afectaría indudablemente el sistema vascular extraóseo. El limitado flujo sanguíneo de la rótula después de la cirugía sería responsable de malos resultados que conducen a dolor en cara anterior de la rodilla, osteonecrosis, fracturas por sobrecarga, y aflojamiento de los componentes rotulianos, ${ }^{12}$ todo lo anterior obliga 
a buscar alterativas menos cruentas que en teoría mejoren los resultados postoperatorios.

El abordaje subvasto se caracteriza por ser más anatómico, la disección quirúrgica sigue la anatomía del cuádriceps, desprende al vasto medial oblicuo de su inserción medial sin afectar el espesor del mecanismo extensor y con apego a los principios generales de los abordajes, por lo tanto en teoría no afecta la fuerza muscular del conjunto, la fuerza vectorial centraliza el recorrido patelar reduciendo la presencia de subluxación o luxación de la patela evitando el uso de liberaciones laterales, respetar los intervalos musculares evita el daño completo del anillo anastomótico, limitando el sacrificio vascular de la rótula. Las desventajas se asocian a la limitada visibilidad del compartimento lateral, por lo que no puede utilizarse en cirugía de revisión, deformidades severas, osteotomías previas, pacientes obesos o musculosos. La disección debe ser cuidadosa para evitar lesionar las estructuras vasculares del canal aductor.

Un punto trascendente en la evolución postoperatoria y que desde el punto de vista clínico ofrece menos dolor postoperatorio independiente del abordaje a utilizar es el uso de torniquetes, ya que es una práctica que los cirujanos ortopédicos han tratado de soslayar y en la actualidad se ha convertido en un tema controvertido. ${ }^{13}$ En este estudio en ninguna cirugía se aplicó manguito neumático.

\section{Conclusiones}

El abordaje subvasto es una alternativa efectiva para el reemplazo total de rodilla primario. Cualquier cirujano que realice de manera habitual el reemplazo total de rodilla con el abordaje parapatelar medial lo hará con seguridad, ya que es fiable, reproducible y eficaz. A pesar de que el abordaje SV ofrece un mejor rango de movilidad inicial y menos dolor, estos beneficios no son significativos en comparación con el abordaje parapatelar medial, si los contrastamos a largo plazo.

\section{Referencias}

1. Niemelalnen MJ, MaKela KT, Robertsson O, W-Dahl A, Furnes O, Fenstad AM, et al. Different incidences of knee arthroplasty in the Nordic countries. Acta Orthop. 2017; 88 (2): 173-178.

2. Scott CE, Howie CR, MacDonald D, Biant LC. Predicting dissatisfaction following total knee replacement: a prospective study of 1217 patients. J Bone Joint Surg Br. 2010; 92 (9): 1253-1258.

3. Sastre S, Sanchez MD, Lozano L, Orient F, Fontg F, Nuñez M. Total knee arthroplasty: better short-term results after subvastus approach: a randomized, controlled study. Knee Surg Sports Traumatol Arthrosc. 2009; 17 (10): 1184-1188.

4. Scuderi GR, Tenholder M, Capeci C. Surgical approaches in mini-incision total knee arthroplasty. Clin Orthop Relat Res. 2004; (428): 61-67.

5. Weinhardt C, Barisic M, Bergmann EG, Heller KD. Early results of subvastus versus medial parapatellar approach in primary total knee arthroplasty. Arch Orthop Trauma Surg. 2004; 124 (6): 401-403.

6. Cila E, Güzel V, Ozalay M, Tan J, Simsek SA, Kanatli U, et al. Subvastus versus medial parapatellar approach in total knee arthroplasty. Arch Orthop Trauma Surg. 2002; 122 (2): 65-68.

7. Hofmann AA, Plaster RL, Murdock LE. Subvastus (Southern) approach for primary total knee arthroplasty. Clin Orthop Relat Res. 1991; (269): 70-77.

8. Berstock JR, Murray JR, Whitehouse MR, Blom AW, Beswick AD. Medial subvastus versus the medial parapatellar approach for total knee replacement: A systematic review and metaanalysis of randomized controlled trials. EFORT Open Rev. 2018; 3 (3): 78-84.

9. Hubbard JK, Sampson HW, Elledge JR. Prevalence and morphology of the vastus medialis oblique muscle in human cadavers. Anat Rec. 1997; 249 (1): 135-142.

10. Scapinelli R. Blood supply of the human patella. Its relation to ischaemic necrosis after fracture. J Bone Joint Surg Br. 1967; 49 (3): $563-570$

11. DeBell H, Pinter Z, Pinto M, Bergstresser S, Lee S, de Cesar Netto $C$, et al. Vascular supply at risk during lateral release of the patella during total knee arthroplasty: a cadaveric study. J Clin Orthop Trauma. 2019; 10 (1): 107-110.

12. Kirschner MH, Menck J, Nerlich A, Walser R, Bühren V, Hofmann $\mathrm{GO}$. The arterial blood supply of the human patella. Its clinical importance for the operating technique in vascularized knee joint transplantations. Surg Radiol Anat. 1997;19 (6): 345-351.

13. Arthur JR, Spangehl MJ. Tourniquet use in total knee arthroplasty. J Knee Surg. 2019; 32 (8): 719-729.

\section{Conflicto de intereses}

No existe conflicto de intereses. 